\title{
On geodesic strongly $E$-convex sets and geodesic strongly $E$-convex functions
}

\section{Adem Kılıçman* and Wedad Saleh}

\section{"Correspondence:}

akilic@upm.edu.my

Department of Mathematics,

University Putra Malaysia, Serdang,

Malaysia

\begin{abstract}
In this article, geodesic $E$-convex sets and geodesic $E$-convex functions on a Riemannian manifold are extended to the so-called geodesic strongly $E$-convex sets and geodesic strongly $E$-convex functions. Some properties of geodesic strongly E-convex sets are also discussed. The results obtained in this article may inspire future research in convex analysis and related optimization fields.
\end{abstract}

MSC: 52A20; 52A41; 53C20; 53C22

Keywords: geodesic E-convex sets; geodesic E-convex functions; Riemannian manifolds

\section{Introduction}

Convexity and its generalizations play an important role in optimization theory, convex analysis, Minkowski space, and fractal mathematics [1-7]. In order to extend the validity of their results to large classes of optimization, these concepts have been generalized and extended in several directions using novel and innovative techniques. Youness [8] defined $E$-convex sets and $E$-convex functions, which have some important applications in various branches of mathematical sciences [9-11]. However, some results given by Youness [8] seem to be incorrect according to Yang [12]. Chen [13] extended E-convexity to a semi- $E$-convexity and discussed some of there properties. Also, Youness and Emam [14] discussed a new class functions which is called strongly $E$-convex functions by taking the images of two points $x_{1}$ and $x_{2}$ under an operator $E: \mathbb{R}^{n} \rightarrow \mathbb{R}^{n}$ besides the two points themselves. Strong $E$-convexity was extended to a semi-strong $E$-convexity as well as quasi- and pseudo-semi-strong $E$-convexity in [15]. The authors investigated the characterization of efficient solutions for multi-objective programming problems involving semi-strong $E$-convexity [16].

A generalization of convexity on Riemannian manifolds was proposed by Rapcsak [17] and Udriste [18]. Moreover, Iqbal et al. [19] introduced geodesic E-convex sets and geodesic $E$-convex functions on Riemannian manifolds.

Motivated by earlier research works $[18,20-25]$ and by the importance of the concepts of convexity and generalized convexity, we discuss a new class of sets on Riemannian manifolds and a new class of functions defined on them, which are called geodesic strongly $E$-convex sets and geodesic strongly $E$-convex functions, and some of their properties are presented.

(c) 2015 Kılıçman and Saleh. This article is distributed under the terms of the Creative Commons Attribution 4.0 International License (http://creativecommons.org/licenses/by/4.0/), which permits unrestricted use, distribution, and reproduction in any medium, provided you give appropriate credit to the original author(s) and the source, provide a link to the Creative Commons license, and indicate if changes were made. 


\section{Preliminaries}

In this section, we introduce some definitions and well-known results of Riemannian manifolds, which help us throughout the article. We refer to [18] for the standard material on differential geometry.

Let $N$ be a $C^{\infty} m$-dimensional Riemannian manifold, and $T_{z} N$ be the tangent space to $N$ at $z$. Also, assume that $\mu_{z}\left(x_{1}, x_{2}\right)$ is a positive inner product on the tangent space $T_{z} N$ $\left(x_{1}, x_{2} \in T_{z} N\right)$, which is given for each point of $N$. Then a $C^{\infty}$ map $\mu: z \rightarrow \mu_{z}$, which assigns a positive inner product $\mu_{z}$ to $T_{z} N$ for each point $z$ of $N$ is called a Riemannian metric.

The length of a piecewise $C^{1}$ curve $\eta:\left[a_{1}, a_{2}\right] \rightarrow N$ which is defined as follows:

$$
L(\eta)=\int_{a_{1}}^{a_{2}}\|\dot{\eta}(x)\| d x
$$

We define $d\left(z_{1}, z_{2}\right)=\inf \left\{L(\eta): \eta\right.$ is a piecewise $C^{1}$ curve joining $z_{1}$ to $\left.z_{2}\right\}$ for any points $z_{1}, z_{2} \in N$. Then $d$ is a distance which induces the original topology on $N$. As we know on every Riemannian manifold there is a unique determined Riemannian connection, called a Levi-Civita connection, denoted by $\nabla_{X} Y$, for any vector fields $X, Y \in N$. Also, a smooth path $\eta$ is a geodesic if and only if its tangent vector is a parallel vector field along the path $\eta$, i.e., $\eta$ satisfies the equation $\nabla \dot{\eta}(t) \eta(t)=0$. Any path $\eta$ joining $z_{1}$ and $z_{2}$ in $N$ such that $L(\eta)=d\left(z_{1}, z_{2}\right)$ is a geodesic and is called a minimal geodesic.

Finally, assume that $(N, \eta)$ is a complete $m$-dimensional Riemannian manifold with Riemannian connection $\nabla$. Let $x_{1}, x_{2} \in N$ and $\eta:[0,1] \rightarrow N$ be a geodesic joining the points $x_{1}$ and $x_{2}$, which means that $\eta_{x_{1}, x_{2}}(0)=x_{2}$ and $\eta_{x_{1}, x_{2}}(1)=x_{1}$.

Definition 2.1 [18] A set $B$ in a Riemannian manifold $N$ is called totally convex if $B$ contains every geodesic $\eta_{x_{1}, x_{2}}$ of $N$ whose endpoints $x_{1}$ and $x_{2}$ belong to $B$.

Note the whole of the manifold $N$ is totally convex, and conventionally, so is the empty set. The minimal circle in a hyperboloid is totally convex, but a single point is not. Also, any proper subset of a sphere is not necessarily totally convex.

The following theorem was proved in [18].

Theorem 2.2 [18] The intersection of any number of a totally convex sets is totally convex.

Remark 2.3 In general, the union of a totally convex set is not necessarily totally convex.

Definition 2.4 [18] A function $f: B \rightarrow \mathbb{R}$ is called a geodesic convex function on atally convex set $B \subset N$ if for every geodesic $\eta_{x_{1}, x_{2}}$, then

$$
f\left(\eta_{x_{1}, x_{2}}(\gamma)\right) \leq \gamma f\left(x_{1}\right)+(1-\gamma) f\left(x_{2}\right)
$$

holds for all $x_{1}, x_{2} \in B$ and $\gamma \in[0,1]$.

In 2005, strongly $E$-convex sets and strongly $E$-convex functions were introduced by Youness and Emam [14] as follows. 


\section{Definition 2.5 [14]}

(1) A subset $B \subseteq \mathbb{R}^{n}$ is called a strongly $E$-convex set if there is a map $E: \mathbb{R}^{n} \rightarrow \mathbb{R}^{n}$ such that

$$
\gamma\left(\alpha b_{1}+E\left(b_{1}\right)\right)+(1-\gamma)\left(\alpha b_{2}+E\left(b_{2}\right)\right) \in B
$$

for each $b_{1}, b_{2} \in B, \alpha \in[0,1]$ and $\gamma \in[0,1]$.

(2) A function $f: B \subseteq \mathbb{R}^{n} \rightarrow \mathbb{R}$ is called a strongly $E$-convex function on $N$ if there is a map $E: \mathbb{R}^{n} \rightarrow \mathbb{R}^{n}$ such that $B$ is a strongly $E$-convex set and

$$
f\left(\gamma\left(\alpha b_{1}+E\left(b_{1}\right)\right)+(1-\gamma)\left(\alpha b_{2}+E\left(b_{2}\right)\right)\right) \leq \gamma f\left(E\left(b_{1}\right)\right)+(1-\gamma) f\left(E\left(b_{2}\right)\right)
$$

for each $b_{1}, b_{2} \in B, \alpha \in[0,1]$ and $\gamma \in[0,1]$.

In 2012, the geodesic $E$-convex set and geodesic $E$-convex functions on a Riemannian manifold were introduced by Iqbal et al. [19] as follows.

\section{Definition 2.6 [19]}

(1) Assume that $E: N \rightarrow N$ is a map. A subset $B$ in a Riemannian manifold $N$ is called geodesic $E$-convex iff there exists a unique geodesic $\eta_{E\left(b_{1}\right), E\left(b_{2}\right)}(\gamma)$ of length $d\left(b_{1}, b_{2}\right)$, which belongs to $B$, for each $b_{1}, b_{2} \in B$ and $\gamma \in[0,1]$.

(2) A function $f: B \subseteq N \rightarrow \mathbb{R}$ is called geodesic $E$-convex on a geodesic $E$-convex $\operatorname{set} B$ if

$$
f\left(\eta_{E\left(b_{1}\right), E\left(b_{2}\right)}(\gamma)\right) \leq \gamma f\left(E\left(b_{1}\right)\right)+(1-\gamma) f\left(E\left(b_{2}\right)\right)
$$

for all $b_{1}, b_{2} \in B$ and $\gamma \in[0,1]$.

\section{Geodesic strongly $E$-convex sets and geodesic strongly $E$-convex functions}

In this section, we introduce a geodesic strongly $E$-convex (GSEC) set and a geodesic strongly $E$-convex (GSEC) function in a Riemannian manifold $N$ and discuss some of their properties.

Definition 3.1 Assume that $E: N \rightarrow N$ is a map. A subset $B$ in a Riemannian manifold $N$ is called GSEC if and only if there is a unique geodesic $\eta_{\alpha b_{1}+E\left(b_{1}\right), \alpha b_{2}+E\left(b_{2}\right)}(\gamma)$ of length $d\left(b_{1}, b_{2}\right)$, which belongs to $B, \forall b_{1}, b_{2} \in B, \alpha \in[0,1]$, and $\gamma \in[0,1]$.

\section{Remark 3.2}

(1) Every GSEC set is a GEC set when $\alpha=0$.

(2) A GEC set is not necessarily a GSEC set. The following example shows this statement.

Example 3.3 Let $N^{2}$ be a 2-dimensional simply complete Riemannian manifold of nonpositive sectional curvature, and $B \subset N^{2}$ be an open star-shaped. Let $E: N^{2} \rightarrow N^{2}$ be a map such that $E(z)=\{y: y \in \operatorname{ker}(B), \forall z \in B\}$. Then $B$ is GEC; on the other hand it is not GSEC. 
Proposition 3.4 Every convex set $B \subset N$ is a GSEC set.

Proof Let us take a map $E: N \rightarrow N$ such as $E=I$ where $I$ is the identity map and $\alpha=0$, then we have the required result.

Note if we take the mapping $E(x)=(1-\alpha) x, x \in B$, then the definition of a GSE reduces to the definition of a $t$-convex set.

Theorem 3.5 If $B \subset N$ is a GSEC set, then $E(B) \subseteq B$.

Proof Since $B$ is a GSEC set, we have for each $b_{1}, b_{2} \in B, \alpha \in[0,1]$, and $\gamma \in[0,1]$,

$$
\eta_{\alpha b_{1}+E\left(b_{1}\right), \alpha b_{2}+E\left(b_{2}\right)}(\gamma) \in B
$$

For $\gamma=0$ and $\alpha=0$, we have $\eta_{E\left(b_{1}\right), E\left(b_{2}\right)}(0)=E\left(b_{2}\right) \in B$, then $E(B) \subseteq B$.

Theorem 3.6 If $\left\{B_{j}, j \in I\right\}$ is an arbitrary family of GSEC subsets of $N$ with respect to the mapping $E: N \rightarrow N$, then the intersection $\bigcap_{j \in I} B_{j}$ is a GSEC subset of $N$.

Proof If $\bigcap_{j \in I} B_{j}$ is an empty set, then it is obviously a GSEC subset of $N$. Assume that $b_{1}, b_{2} \in \bigcap_{j \in I} B_{j}$, then $b_{1}, b_{2} \in B_{j}, \forall j \in I$. By the GSEC of $B_{j}$, we get $\eta_{\alpha b_{1}+E\left(b_{1}\right), \alpha b_{2}+E\left(b_{2}\right)}(\gamma) \in B_{j}$, $\forall j \in I, \alpha \in[0,1]$, and $\gamma \in[0,1]$. Hence, $\eta_{\alpha b_{1}+E\left(b_{1}\right), \alpha b_{2}+E\left(b_{2}\right)}(\gamma) \in \bigcap_{j \in I} B_{j}, \forall \alpha \in[0,1]$ and $\gamma \in$ $[0,1]$.

Remark 3.7 The above theorem is not generally true for the union of GSEC subsets of $N$.

Now, we extend the definition of a GEC function on a Riemannian manifold to a GSEC function on a Riemannian manifold.

Definition 3.8 A real-valued function $f: B \subset N \rightarrow \mathbb{R}$ is said to be a GSEC function on a GSEC set $B$, if

$$
f\left(\eta_{\alpha b_{1}+E\left(b_{1}\right), \alpha b_{2}+E\left(b_{2}\right)}(\gamma)\right) \leq \gamma f\left(E\left(b_{1}\right)\right)+(1-\gamma) f\left(E\left(b_{2}\right)\right),
$$

$\forall b_{1}, b_{2} \in B, \alpha \in[0,1]$, and $\gamma \in[0,1]$. If the above inequality is strict for all $b_{1}, b_{2} \in B, \alpha b_{1}+$ $E\left(b_{1}\right) \neq \alpha b_{2}+E\left(b_{2}\right), \alpha \in[0,1]$, and $\gamma \in(0,1)$, then $f$ is called a strictly GSEC function.

\section{Remark 3.9}

(1) Every GSEC function is a GEC function when $\alpha=0$. The following example shows that a GEC function is not necessarily a GSEC function.

Example 3.10 Consider the function $f: \mathbb{R} \rightarrow \mathbb{R}$ where $f(b)=-|b|$ and suppose that $E: \mathbb{R} \rightarrow \mathbb{R}$ is given as $E(b)=-b$. We consider the geodesic $\eta$ such that

$$
\begin{aligned}
\eta_{\alpha b_{1}+E\left(b_{1}\right), \alpha b_{2}+E\left(b_{2}\right)}(\gamma) & = \begin{cases}-\left[\alpha b_{2}+E\left(b_{2}\right)+\gamma\left(\alpha b_{1}+E\left(b_{1}\right)-\alpha b_{2}-E\left(b_{2}\right)\right)\right] ; & b_{1} b_{2} \geq 0, \\
-\left[\alpha b_{2}+E\left(b_{2}\right)+\gamma\left(\alpha b_{2}+E\left(b_{2}\right)-\alpha b_{1}-E\left(b_{1}\right)\right)\right] ; & b_{1} b_{2}<0\end{cases} \\
& = \begin{cases}-\left[(\alpha-1) b_{2}+\gamma\left((\alpha-1) b_{1}+(1-\alpha) b_{2}\right)\right] ; & b_{1} b_{2} \geq 0, \\
-\left[(\alpha-1) b_{2}+\gamma\left((\alpha-1) b_{2}+(1-\alpha) b_{1}\right)\right] ; & b_{1} b_{2}<0 .\end{cases}
\end{aligned}
$$


If $\alpha=0$, then

$$
\eta_{E\left(b_{1}\right), E\left(b_{2}\right)}(\gamma)= \begin{cases}{\left[b_{2}+\gamma\left(b_{1}-b_{2}\right)\right] ;} & b_{1} b_{2} \geq 0 \\ {\left[b_{2}+\gamma\left(b_{2}-b_{1}\right)\right] ;} & b_{1} b_{2}<0\end{cases}
$$

If $b_{1}, b_{2} \geq 0$, then

$$
\begin{aligned}
f\left(\eta_{E\left(b_{1}\right), E\left(b_{2}\right)}(\gamma)\right) & =f\left(b_{2}+\gamma\left(b_{1}-b_{2}\right)\right) \\
& =-\left[(1-\gamma) b_{2}+\gamma b_{1}\right] .
\end{aligned}
$$

On the other hand

$$
\gamma f\left(E\left(b_{1}\right)\right)+(1-\gamma) f\left(E\left(b_{2}\right)\right)=\gamma f\left(-b_{1}\right)+(1-\gamma) f\left(-b_{2}\right)=-\left[(1-\gamma) b_{2}+\gamma b_{1}\right] .
$$

Hence, $f\left(\eta_{E\left(b_{1}\right), E\left(b_{2}\right)}(\gamma)\right) \leq \gamma f\left(E\left(b_{1}\right)\right)+(1-\gamma) f\left(E\left(b_{2}\right)\right), \forall \gamma \in[0,1]$.

Similarly, the above inequality holds true when $b_{1}, b_{2}<0$.

Now, let $b_{1}<0, b_{2}>0$, then

$$
\begin{aligned}
f\left(\eta_{E\left(b_{1}\right), E\left(b_{2}\right)}(\gamma)\right) & =f\left(b_{2}+\gamma\left(b_{2}-b_{1}\right)\right) \\
& =-\left[(1+\gamma) b_{2}-\gamma b_{1}\right] .
\end{aligned}
$$

On the other hand

$$
\gamma f\left(E\left(b_{1}\right)\right)+(1-\gamma) f\left(E\left(b_{2}\right)\right)=\gamma f\left(-b_{1}\right)+(1-\gamma) f\left(-b_{2}\right)=\gamma b_{1}-(1-\gamma) b_{2} .
$$

It follows that

$$
f\left(\eta_{E\left(b_{1}\right), E\left(b_{2}\right)}(\gamma)\right) \leq \gamma f\left(E\left(b_{1}\right)\right)+(1-\gamma) f\left(E\left(b_{2}\right)\right)
$$

if and only if

$$
-\left[(1+\gamma) b_{2}-\gamma b_{1}\right] \leq \gamma b_{1}-(1-\gamma) b_{2}
$$

if and only if

$$
-2 \gamma b_{2} \leq 0
$$

which is always true for all $\gamma \in[0,1]$.

Similarly, $f\left(\eta_{E\left(b_{1}\right), E\left(b_{2}\right)}(\gamma)\right) \leq \gamma f\left(E\left(b_{1}\right)\right)+(1-\gamma) f\left(E\left(b_{2}\right)\right), \forall \gamma \in[0,1]$ also holds for $b_{1}>0$ and $b_{2}<0$.

Thus, $f$ is a GEC function on $\mathbb{R}$, but it is not a GSEC function because if we take $b_{1}=0$, $b_{2}=-1$ and $\gamma=\frac{1}{2}$, then

$$
\begin{aligned}
f\left(\eta_{\alpha b_{1}+E\left(b_{1}\right), \alpha b_{2}+E\left(b_{2}\right)}(\gamma)\right) & =f\left(\frac{1}{2} \alpha-\frac{1}{2}\right) \\
& =\frac{1}{2} \alpha-\frac{1}{2}
\end{aligned}
$$




$$
\begin{aligned}
& >\frac{1}{2} f(E(0))+\frac{1}{2} f(E(-1)) \\
& =\frac{-1}{2}, \quad \forall \alpha \in(0,1] .
\end{aligned}
$$

(2) Every $g$-convex function $f$ on a convex set $B$ is a GSEC function when $\alpha=0$ and $E$ is the identity map.

Proposition 3.11 Assume that $f: B \rightarrow \mathbb{R}$ is a GSEC function on a GSEC set $B \subseteq N$, then $f(\alpha b+E(b)) \leq f(E(b)), \forall b \in B$ and $\alpha \in[0,1]$.

Proof Since $f: B \rightarrow \mathbb{R}$ is a GSEC function on a GSEC set $B \subseteq N$, then $\eta_{\alpha b_{1}+E\left(b_{1}\right), \alpha b_{2}+E\left(b_{2}\right)}(\gamma) \in$ $B, \forall b_{1}, b_{2} \in B, \alpha \in[0,1]$, and $\gamma \in[0,1]$. Also,

$$
f\left(\eta_{\alpha b_{1}+E\left(b_{1}\right), \alpha b_{2}+E\left(b_{2}\right)}(\gamma)\right) \leq \gamma f\left(E\left(b_{1}\right)\right)+(1-\gamma) f\left(E\left(b_{2}\right)\right)
$$

thus, for $\gamma=1$, we get $\eta_{\alpha b_{1}+E\left(b_{1}\right), \alpha b_{2}+E\left(b_{2}\right)}(\gamma)=\alpha b_{1}+E\left(b_{1}\right)$. Then

$$
f\left(\alpha b_{1}+E\left(b_{1}\right)\right) \leq f\left(E\left(b_{1}\right)\right)
$$

Theorem 3.12 Consider that $B \subseteq N$ is a GSEC set and $f_{1}: B \rightarrow \mathbb{R}$ is a GSEC function. If $f_{2}: I \rightarrow \mathbb{R}$ is a non-decreasing convex function such that $\operatorname{rang}\left(f_{1}\right) \subset I$, then $f_{2} \circ f_{1}$ is a GSEC function on $B$.

Proof Since $f_{1}$ is a GSEC function, for all $b_{1}, b_{2} \in B, \alpha \in[0,1]$, and $\gamma \in[0,1]$,

$$
f_{1}\left(\eta_{\alpha b_{1}+E\left(b_{1}\right), \alpha b_{2}+E\left(b_{2}\right)}(\gamma)\right) \leq \gamma f_{1}\left(E\left(b_{1}\right)\right)+(1-\gamma) f_{1}\left(E\left(b_{2}\right)\right) .
$$

Since $f_{2}$ is a non-decreasing convex function,

$$
\begin{aligned}
f_{2} & \circ f_{2}\left(\eta_{\alpha b_{1}+E\left(b_{1}\right), \alpha b_{2}+E\left(b_{2}\right)}(\gamma)\right) \\
& =f_{2}\left(f_{2}\left(\eta_{\alpha b_{1}+E\left(b_{1}\right), \alpha b_{2}+E\left(b_{2}\right)}(\gamma)\right)\right) \\
& \leq f_{2}\left(\gamma f_{1}\left(E\left(b_{1}\right)\right)+(1-\gamma) f_{1}\left(E\left(b_{2}\right)\right)\right) \\
& \leq \gamma f_{2}\left(f_{1}\left(E\left(b_{1}\right)\right)\right)+(1-\gamma) f_{2}\left(f_{1}\left(E\left(b_{2}\right)\right)\right) \\
& =\gamma\left(f_{2} \circ f_{1}\right)\left(E\left(b_{1}\right)\right)+(1-\gamma)\left(f_{2} \circ f_{1}\right)\left(E\left(b_{2}\right)\right),
\end{aligned}
$$

which means that $f_{2} \circ f_{1}$ is a GSEC function on $B$. Similarly, if $f_{2}$ is a strictly non-decreasing convex function, then $f_{2} \circ f_{1}$ is a strictly GSEC function.

Theorem 3.13 Assume that $B \subseteq N$ is a GSEC set and $f_{j}: B \rightarrow \mathbb{R}, j=1,2, \ldots, m$ are GSEC functions. Then the function

$$
f=\sum_{j=1}^{m} n_{j} f_{j}
$$

is GSEC on $B, \forall n_{j} \in \mathbb{R}, n_{j} \geq 0$. 
Proof Since $f_{j}, j=1,2, \ldots, m$ are GSEC functions, $\forall b_{1}, b_{2} \in B, \alpha \in[0,1]$, and $\gamma \in[0,1]$, we have

$$
f_{j}\left(\eta_{\alpha b_{1}+E\left(b_{1}\right), \alpha b_{2}+E\left(b_{2}\right)}(\gamma)\right) \leq \gamma f_{j}\left(E\left(b_{1}\right)\right)+(1-\gamma) f_{j}\left(E\left(b_{2}\right)\right) .
$$

It follows that

$$
n_{j} f_{j}\left(\eta_{\alpha b_{1}+E\left(b_{1}\right), \alpha b_{2}+E\left(b_{2}\right)}(\gamma)\right) \leq \gamma n_{j} f_{j}\left(E\left(b_{1}\right)\right)+(1-\gamma) n_{j} f_{j}\left(E\left(b_{2}\right)\right) .
$$

Then

$$
\begin{aligned}
& \sum_{j=1}^{m} n_{j} f_{j}\left(\eta_{\alpha b_{1}+E\left(b_{1}\right), \alpha b_{2}+E\left(b_{2}\right)}(\gamma)\right) \\
& \quad \leq \gamma \sum_{j=1}^{m} n_{j} f_{j}\left(E\left(b_{1}\right)\right)+(1-\gamma) \sum_{j=1}^{m} n_{j} f_{j}\left(E\left(b_{2}\right)\right) \\
& \quad=\gamma f\left(E\left(b_{1}\right)\right)+(1-\gamma) f\left(E\left(b_{2}\right)\right) .
\end{aligned}
$$

Thus, $f$ is a GSEC function.

Theorem 3.14 Let $B \subseteq N$ be a GSEC set and $\left\{f_{j}, j \in I\right\}$ be a family of real-valued functions defined on $B$ such that $\sup _{j \in I} f_{j}(b)$ exists in $\mathbb{R}, \forall b \in B$. If $f_{j}: B \rightarrow \mathbb{R}, j \in I$ are GSEC functions on $B$, then the function $f: B \rightarrow \mathbb{R}$, defined by $f(b)=\sup _{j \in I} f_{j}(b), \forall b \in B$ is GSEC on $B$.

Proof Since $f_{j}, j \in I$ are GSEC functions on a GSEC set $B, \forall b_{1}, b_{2} \in B, \alpha \in[0,1]$, and $\gamma \in$ $[0,1]$, we have

$$
f_{j}\left(\eta_{\alpha b_{1}+E\left(b_{1}\right), \alpha b_{2}+E\left(b_{2}\right)}(\gamma)\right) \leq \gamma f_{j}\left(E\left(b_{1}\right)\right)+(1-\gamma) f_{j}\left(E\left(b_{2}\right)\right) .
$$

Then

$$
\begin{aligned}
& \sup _{j \in I} f_{j}\left(\eta_{\alpha b_{1}+E\left(b_{1}\right), \alpha b_{2}+E\left(b_{2}\right)}(\gamma)\right) \\
& \quad \leq \sup _{j \in I}\left[\gamma f_{j}\left(E\left(b_{1}\right)\right)+(1-\gamma) f_{j}\left(E\left(b_{2}\right)\right)\right] \\
& \quad=\gamma \sup _{j \in I} f_{j}\left(E\left(b_{1}\right)\right)+(1-\gamma) \sup _{j \in I} f_{j}\left(E\left(b_{2}\right)\right) \\
& \quad=\gamma f\left(E\left(b_{1}\right)\right)+(1-\gamma) f\left(E\left(b_{2}\right)\right) .
\end{aligned}
$$

Hence,

$$
f\left(\eta_{\alpha b_{1}+E\left(b_{1}\right), \alpha b_{2}+E\left(b_{2}\right)}(\gamma)\right) \leq \gamma f\left(E\left(b_{1}\right)\right)+(1-\gamma) f\left(E\left(b_{2}\right)\right),
$$

which means that $f$ is a GSEC function on $B$.

Proposition 3.15 Assume that $h_{j}: N \rightarrow \mathbb{R}, j=1,2, \ldots, m$ are GSEC functions on $N$, with respect to $E: N \rightarrow N$. If $E(B) \subseteq B$, then $B=\left\{b \in N: h_{j}(b) \leq 0, j=1,2, \ldots, m\right\}$ is a GSEC set. 
Proof Since $h_{j}, j=1,2, \ldots m$ are GSEC functions,

$$
\begin{aligned}
h_{j}\left(\eta_{\alpha b_{1}+E\left(b_{1}\right), \alpha b_{2}+E\left(b_{2}\right)}(\gamma)\right) & \leq \gamma h_{j}\left(E\left(b_{1}\right)\right)+(1-\gamma) h_{j}\left(E\left(b_{2}\right)\right) \\
& \leq 0
\end{aligned}
$$

$\forall b_{1}, b_{2} \in B, \alpha \in[0,1]$, and $\gamma \in[0,1]$. Since $E(B) \subseteq B, \eta_{\alpha b_{1}+E\left(b_{1}\right), \alpha b_{2}+E\left(b_{2}\right)}(\gamma) \in B$. Hence, $B$ is a GSEC set.

\section{Epigraphs}

Youness and Emam [14] defined a strongly $E \times F$-convex set where $E: \mathbb{R}^{n} \rightarrow \mathbb{R}^{n}$ and $F: \mathbb{R} \rightarrow \mathbb{R}$ and studied some of its properties. In this section, we generalize a strongly $E \times F$-convex set to a geodesic strongly $E \times F$-convex set on Riemannian manifolds and discuss GSEC functions in terms of their epigraphs. Furthermore, some properties of GSE sets are given.

Definition 4.1 Let $B \subset N \times \mathbb{R}, E: N \rightarrow N$ and $F: \mathbb{R} \rightarrow \mathbb{R}$. A set $B$ is called geodesic strongly $E \times F$-convex if $\left(b_{1}, \beta_{1}\right),\left(b_{2}, \beta_{2}\right) \in B$ implies

$$
\left(\eta_{\alpha b_{1}+E\left(b_{1}\right), \alpha b_{2}+E\left(b_{2}\right)}(\gamma), \gamma F\left(\beta_{1}\right)+(1-\gamma) F\left(\beta_{2}\right)\right) \in B
$$

for all $\alpha \in[0,1]$ and $\gamma \in[0,1]$.

It is not difficult to prove that $B \subseteq N$ is a GSEC set if and only if $B \times \mathbb{R}$ is a geodesic strongly $E \times F$-convex set.

An epigraph of $f$ is given by

$$
\operatorname{epi}(f)=\{(b, a): b \in B, a \in \mathbb{R}, f(b) \leq a\} .
$$

A characterization of a GSEC function in terms of its epi $(f)$ is given by the following theorem.

Theorem 4.2 Let $E: N \rightarrow N$ be a map, $B \subseteq N$ be a GSEC set, $f: B \rightarrow \mathbb{R}$ be a real-valued function and $F: \mathbb{R} \rightarrow \mathbb{R}$ be a map such that $F(f(b)+a)=f(E(b))+a$, for each non-negative real number a. Then $f$ is a GSEC function on $B$ if and only if epi $(f)$ is geodesic strongly $E \times F$-convex on $B \times \mathbb{R}$.

Proof Assume that $\left(b_{1}, a_{1}\right),\left(b_{2}, a_{2}\right) \in \operatorname{epi}(f)$. If $B$ is a GSEC set, then $\eta_{\alpha b_{1}+E\left(b_{1}\right), \alpha b_{2}+E\left(b_{2}\right)}(\gamma) \in$ $B, \forall \alpha \in[0,1]$ and $\gamma \in[0,1]$. Since $E\left(b_{1}\right) \in B$ for $\alpha=0, \gamma=1$, also $E\left(b_{2}\right) \in B$ for $\alpha=0$, $\gamma=0$, let $F\left(a_{1}\right)$ and $F\left(a_{2}\right)$ be such that $f\left(E\left(b_{1}\right)\right) \leq F\left(a_{1}\right)$ and $f\left(E\left(b_{2}\right)\right) \leq F\left(a_{2}\right)$. Then $\left(E\left(b_{1}\right), F\left(a_{1}\right)\right),\left(E\left(b_{2}\right), F\left(a_{2}\right)\right) \in \operatorname{epi}(f)$.

Let $f$ be GSEC on $B$, then

$$
\begin{aligned}
f\left(\eta_{\alpha b_{1}+E\left(b_{1}\right), \alpha b_{2}+E\left(b_{2}\right)}(\gamma)\right) & \leq \gamma f\left(E\left(b_{1}\right)\right)+(1-\gamma) f\left(E\left(b_{2}\right)\right) \\
& \leq \gamma F\left(a_{1}\right)+(1-\gamma) F\left(a_{2}\right) .
\end{aligned}
$$

Thus, $\left(\eta_{\alpha b_{1}+E\left(b_{1}\right), \alpha b_{2}+E\left(b_{2}\right)}(\gamma), \gamma F\left(a_{1}\right)+(1-\gamma) F\left(a_{2}\right)\right) \in$ epi $(f)$, then epi $(f)$ is geodesic strongly $E \times F$-convex on $B \times \mathbb{R}$. 
Conversely, assume that epi $(f)$ is geodesic strongly $E \times F$-convex on $B \times \mathbb{R}$. Let $b_{1}, b_{2} \in B$, $\alpha \in[0,1]$, and $\gamma \in[0,1]$, then $\left(b_{1}, f\left(b_{1}\right)\right) \in \operatorname{epi}(f)$ and $\left(b_{2}, f\left(b_{2}\right)\right) \in \operatorname{epi}(f)$. Now, since epi $(f)$ is geodesic strongly $E \times F$-convex on $B \times \mathbb{R}$, we obtain $\left(\eta_{\alpha b_{1}+E\left(b_{1}\right), \alpha b_{2}+E\left(b_{2}\right)}(\gamma), \gamma F\left(f\left(b_{1}\right)\right)+\right.$ $\left.(1-\gamma) F\left(f\left(b_{2}\right)\right)\right) \in \operatorname{epi}(f)$, then

$$
\begin{aligned}
f\left(\eta_{\alpha b_{1}+E\left(b_{1}\right), \alpha b_{2}+E\left(b_{2}\right)}(\gamma)\right) & \leq \gamma F\left(f\left(b_{1}\right)\right)+(1-\gamma) F\left(f\left(b_{2}\right)\right) \\
& =\gamma f\left(E\left(b_{1}\right)\right)+(1-\gamma) f\left(E\left(b_{2}\right)\right) .
\end{aligned}
$$

This shows that $f$ is a GSEC function on $B$.

Theorem 4.3 Assume that $\left\{B_{j}, j \in I\right\}$ is a family of geodesic strongly $E \times F$-convex sets. Then the intersection $\bigcap_{j \in I} B_{j}$ is a geodesic strongly $E \times F$-convex set.

Proof Assume that $\left(b_{1}, a_{1}\right),\left(b_{2}, a_{2}\right) \in \bigcap_{j \in I} B_{j}$, so $\forall j \in I,\left(b_{1}, a_{1}\right),\left(b_{2}, a_{2}\right) \in B_{j}$. Since $B_{j}$ is the geodesic strongly $E \times F$-convex sets $\forall j \in I$, we have

$$
\left(\eta_{\alpha b_{1}+E\left(b_{1}\right), \alpha b_{2}+E\left(b_{2}\right)}(\gamma), \gamma F\left(a_{1}\right)+(1-\gamma) F\left(a_{2}\right)\right) \in B_{j}
$$

$\forall \alpha \in[0,1]$ and $\gamma \in[0,1]$. Therefore,

$$
\left(\eta_{\alpha b_{1}+E\left(b_{1}\right), \alpha b_{2}+E\left(b_{2}\right)}(\gamma), \gamma F\left(a_{1}\right)+(1-\gamma) F\left(a_{2}\right)\right) \in \bigcap_{j \in I} B_{j},
$$

$\forall \alpha \in[0,1]$ and $\gamma \in[0,1]$. Then $\bigcap_{j \in I} B_{j}$ is a geodesic strongly $E \times F$-convex set.

Theorem 4.4 Assume that $E: N \rightarrow N$ and $F: \mathbb{R} \rightarrow \mathbb{R}$ are two maps such that $F(f(b)+a)=$ $f(E(b))+$ a for each non-negative real number a. Suppose that $\left\{f_{j}, j \in I\right\}$ is a family of realvalued functions defined on a GSEC set $B \subseteq N$ which are bounded from above. If epi $\left(f_{j}\right)$ are geodesic strongly $E \times F$-convex sets, then the function $f$ which is given by $f(b)=\sup _{j \in I} f_{j}(b)$, $\forall b \in B$, is a GSEC function on $B$.

Proof If each $f_{j}, j \in I$ is a GSEC function on a GSEC geodesic set $B$, then

$$
\operatorname{epi}\left(f_{j}\right)=\left\{(b, a): b \in B, a \in \mathbb{R}, f_{j}(b) \leq a\right\}
$$

are geodesic strongly $E \times F$-convex on $B \times \mathbb{R}$. Therefore,

$$
\begin{aligned}
\bigcap_{j \in I} \operatorname{epi}\left(f_{j}\right) & =\left\{(b, a): b \in B, a \in \mathbb{R}, f_{j}(b) \leq a, j \in I\right\} \\
& =\{(b, a): b \in B, a \in \mathbb{R}, f(b) \leq a\}
\end{aligned}
$$

is geodesic strongly $E \times F$-convex set. Then, according to Theorem 4.2 we see that $f$ is a GSEC function on $B$. 
Authors' contributions

All authors jointly worked on deriving the results and approved the final manuscript.

\section{Acknowledgements}

The authors would like to thank the referees for valuable suggestions and comments, which helped the authors to improve this article substantially.

Received: 1 May 2015 Accepted: 15 September 2015 Published online: 25 September 2015

References

1. Kılıçman, A, Saleh, W: A note on starshaped sets in 2-dimensional manifolds without conjugate points. J. Funct. Spaces 2014, Article ID 675735 (2014)

2. Boltyanski, V, Martini, H, Soltan, PS: Excursions into Combinatorial Geometry. Springer, Berlin (1997)

3. Danzer, L, Grünbaum, B, Klee, V: Helly's theorem and its relatives. In: Klee, V (ed.) Convexity. Proc. Sympos. Pure Math., vol. 7, pp. 101-180 (1963)

4. Jiménez, MA, Garzón, GR, Lizana, AR: Optimality Conditions in Vector Optimization. Bentham Science Publishers, Sharjah (2010)

5. Martini, H, Swanepoel, KJ: Generalized convexity notions and combinatorial geometry. Congr. Numer. 164, 65-93 (2003)

6. Martini, H, Swanepoel, KJ: The geometry of Minkowski spaces - a survey. Part II. Expo. Math. 22, 14-93 (2004)

7. Saleh, W, Kılıçman, A: On generalized s-convex functions on fractal sets. JP J. Geom. Topol. 17(1), 63-82 (2015)

8. Youness, EA: E-Convex sets, E-convex functions and E-convex programming. J. Optim. Theory Appl. 102, 439-450 (1999)

9. Abou-Tair, I, Sulaiman, WT: Inequalities via convex functions. Int. J. Math. Math. Sci. 22(3), 543-546 (1999)

10. Noor, MA: Fuzzy preinvex functions. Fuzzy Sets Syst. 64, 95-104 (1994)

11. Noor, MA, Noor, Kl, Awan, MU: Generalized convexity and integral inequalities. Appl. Math. Inf. Sci. 24(8), 1384-1388 (2015)

12. Yang, X: On E-convex sets, E-convex functions, and E-convex programming. J. Optim. Theory Appl. 109(3), 699-704 (2001)

13. Chen, X: Some properties of semi-E-convex functions. J. Math. Anal. Appl. 275(1), 251-262 (2002)

14. Youness, EA, Emam, T: Strongly E-convex sets and strongly E-convex functions. J. Interdiscip. Math. 8(1), 107-117 (2005)

15. Youness, EA, Emam, T: Semi-strongly E-convex functions. J. Math. Stat. 1(1), 51-57 (2005)

16. Youness, EA, Emam, T: Characterization of efficient solutions for multi-objective optimization problems involving semi-strong and generalized semi-strong E-convexity. Acta Math. Sci., Ser. B Engl. Ed. 28(1), 7-16 (2008)

17. Rapcsak, T: Smooth Nonlinear Optimization in $\mathbb{R}^{n}$. Kluwer Academic, Dordrecht (1997)

18. Udrist, C: Convex Functions and Optimization Methods on Riemannian Manifolds. Kluwer Academic, Dordrecht (1994)

19. Iqbal, A, Ali, S, Ahmad, I: On geodesic E-convex sets, geodesic E-convex functions and E-epigraphs. J. Optim. Theory Appl. 55(1), 239-251 (2012)

20. Fulga, C, Preda, V: Nonlinear programming with E-preinvex and local E-preinvex function. Eur. J. Oper. Res. 192, 737-743 (2009)

21. Iqbal, A, Ahmad, I, Ali, S: Strong geodesic $\alpha$-preinvexity and invariant $\alpha$-monotonicity on Riemannian manifolds. Numer. Funct. Anal. Optim. 31, 1342-1361 (2010)

22. Megahed, AEMA, Gomma, HG, Youness, EA, El-Banna, AZH: Optimality conditions of E-convex programming for an E-differentiable function. J. Inequal. Appl. 2013(1), 246 (2013)

23. Mirzapour, F, Mirzapour, A, Meghdadi, M: Generalization of some important theorems to E-midconvex functions. Appl. Math. Lett. 24(8), 1384-1388 (2011)

24. Syau, YR, Lee, ES: Some properties of E-convex functions. Appl. Math. Lett. 18, 1074-1080 (2005)

25. Yang, XM: On E-convex programming. J. Optim. Theory Appl. 109, 699-704 (2001)

\section{Submit your manuscript to a SpringerOpen ${ }^{\circ}$ journal and benefit from:}

- Convenient online submission

Rigorous peer review

- Immediate publication on acceptance

- Open access: articles freely available online

- High visibility within the field

- Retaining the copyright to your article 